\title{
2-TORSION POINTS ON THETA DIVISORS
}

\author{
GIUSEPPE PARESCHI AND RICCARDO SALVATI MANNI
}

\begin{abstract}
In this note we prove a sharp bound for the number of 2-torsion points on a theta divisor and show that this is achieved only in the case of products of elliptic curves. This settles in the affirmative a conjecture of Marcucci and Pirola.
\end{abstract}

\section{INTRODUCTION}

Let $A$ be a $g$-dimensional principally polarized abelian variety. Let $\Theta$ be a divisor on $A$ representing the principal polarization. We set

$$
\Theta(n):=\# A[n] \cap \Theta,
$$

where $A[n]$ is the group of $n$-torsion points on $A$. The geometry of $\Theta(2)$ is interesting. In fact, once a symmetric divisor $\Theta$ is chosen, $A[2]$ splits in the even and odd part. The odd points lie in $\Theta$. On the other hand, the even ones which lie in $\Theta$ correspond to the classical thetanulls. In the complex case, their presence provides a characterization of decomposable principally polarized abelian variety $([20$, , 4]) or a hyperelliptic jacobian $([15])$.

In [13], Marcucci and Pirola gave a bound for $\Theta(2)$ (over $\mathbb{C}$ ). This bound has been recently improved by Auffarth, Pirola and Salvati Manni in [1] where also a bound for $\Theta(n)$ is given. However, these bounds were not optimal. In 13 it has been conjectured that the maximal $\Theta(2)$ is computed exactly by products of elliptic curves, and a similar conjecture for $\Theta(n)$ has been formulated in [1]. The purpose of this note is to prove the conjecture for $\Theta(2)$. Our methods are algebraic and work in characteristic $\neq 2$. Thus we will characterize principally polarized abelian variety with the maximum number of thetanulls.

Theorem 1.1. Let $A$ be an principally polarized abelian variety over an algebraically closed field of characteristic $\neq 2$. For all divisors $\Theta$ representing the principal polarization

$$
\Theta(2) \leq 4^{g}-3^{g} .
$$

Moreover equality holds if and only if $A$ is a product of elliptic curves and $\Theta$ is a symmetric theta divisor of $A$.

1.1. Acknowledgement. We are grateful to Robert Auffarth and Pietro Pirola for many discussions, suggestions and for their continuous encouragement. The proof of Kempf's Theorem 2.1 outlined in Remark 2.4 originates from work done years ago with Mihnea Popa.

Key words and phrases. abelian variety, theta divisor, torsion.

GP was partially supported by the MIUR Excellence Department Project awarded to the Department of Mathematics, University of Rome Tor Vergata, CUP E83C18000100006". 


\section{Preliminaries}

Let $\Theta$ a symmetric theta divisor representing the principal polarization. All divisors representing the principal polarization are translates of $\Theta$. Let us denote $L=\mathcal{O}_{A}(\Theta)$. We denote $t_{x}: A \rightarrow A$ the translation by $x$. For $x, y \in A$ we consider the multiplication map of global sections

$$
M(x, y): H^{0}\left(A, t_{x}^{*} L^{2}\right) \otimes H^{0}\left(A, t_{y}^{*} L^{2}\right) \rightarrow H^{0}\left(A, t_{x}^{*} L^{2} \otimes t_{y}^{*} L^{2}\right)
$$

Theorem 2.1. (Kempf) The rank of the map $M(x, y)$ is equal to the number of $\eta \in A[2]$ such that

$$
y-x+\eta \notin \Theta
$$

Hence $(\Theta+y)(2)$ is equal to the rank of $M(0, y)$ for all $y \in A$.

This has as a corollary the following

Theorem 2.2. The map $M(x, y)$ is not surjective if and only if

$$
y-x \in \bigcup_{\eta \in A[2]} \Theta+\eta
$$

In particular, for any fixed $x \in A$, the multiplication map $M(x, y)$ is surjective for general $y \in A$

In turn Theorem 2.2 has the following consequence, originally proved by Koizumi ([12])

Theorem 2.3. (Koizumi) For $h, k \geq 2$ and $h+k \geq 5$ the multiplication map of global sections

$$
H^{0}\left(A, t_{x}^{*} L^{h}\right) \otimes H^{0}\left(A, t_{y}^{*} L^{k}\right) \rightarrow H^{0}\left(A, t_{x}^{*} L^{h} \otimes t_{y}^{*} L^{k}\right)
$$

is surjective for all $x, y \in A$.

Theorem 2.3 is well known and admits different proofs (see e.g. [11, Thm 6.8(c)], [17, Example 3.7], [8, Thm D]).

The last assertion of Theorem 2.2 was proved independently by Kempf ([10, Thm 1] and Sekiguchi ([21, Prop. 1.5]). A similar statement, in the more general context of any ample line bundle, was proved independently by Ohbuchi ([16]). A proof not using theta-groups, but rather the Fourier-Mukai transform associated to the Poincaré line bundle, was subsequently given by Pareschi and Popa in [18, Thm 5.8].

The more precise formula of Theorem 2.1 and, consequently, the formula in Theorem 2.2 are again due to $\operatorname{Kempf}([10$, Thm 3]) 1$]$

Remark 2.4. Although Theorem 2.1 is not mentioned in the paper [18], it could have been reproved there within the same Fourier-Mukai methods, by means of an additional argument. For the benefit of readers more familiar with such methods, we do it here. To this purpose in the first place we notice that in Theorem 2.1 it is sufficient to assume that $x=0$. The approach of [17], and subsequently [18, Thm 5.8] identifies the multiplication map $M(0, y)$ with the evaluation at the point $y \in A$ of the global sections of the vector bundle (called "skew Pontryagin product", see [18] Terminology 5.1)) $\mathcal{E}:=L^{2} \hat{*} L^{2}$. Therefore we denote such evaluation map in the same way:

$$
M(0, y): H^{0}(\mathcal{E}) \rightarrow \mathcal{E}(y)
$$

\footnotetext{
${ }^{1} \mathrm{~A}$ word of warning about [10, Thm 3]. There is a small mistake in the statement there: the displayed formula should read: $2 z+\eta \notin \Theta+2 x$ (this is equivalent to the statement of Theorem 2.1 here). In fact there is an inaccuracy in the proof: the beginning of the first line of page 773 should be: $2 s \notin \theta+\eta$.
} 
We recall that the "WIT criterion" given by [18, Thm 4.1] yields that a vector bundle $\mathcal{F}$ is weakly continuously globally generated as soon as the dual vector bundle $\mathcal{F}^{\vee}$ satisfies WIT(g) plus some other technical conditions. In the proof of [18, Thm 5.8] it is shown that the WIT(g) condition (as well as the additional technical conditions) is satisfied by the vector bundle $\left(\mathcal{E} \otimes L^{-1}\right)^{\vee}$ (in the more general context where $L$ can be any ample line bundle on $A$ ). Therefore the vector bundle $\mathcal{E} \otimes L^{-1}$ is weakly continuously globally generated. Moreover in the same proof it is also explicitly computed the Fourier-Mukai transform of the vector bundle $\left(\mathcal{E} \otimes L^{-1}\right)^{\vee}$ (see (1) in the proof of loc.cit.), and it is shown that such calculation implies, in the case at hand, that to be weakly continuously globally generated concretely means that the sum of evaluation maps

$$
\bigoplus_{\alpha \in \operatorname{Pic}^{0} A[2]} H^{0}\left(\mathcal{E} \otimes L^{-1} \otimes \alpha\right) \otimes \alpha \rightarrow \mathcal{E} \otimes L^{-1}
$$

is surjective. Again all this works more generally for any ample line bundle on an abelian variety as well. If $L$ is a principal polarization then $h^{0}\left(\mathcal{E} \otimes L^{-1} \otimes \alpha\right)=1$ for all $\alpha \in \operatorname{Pic}^{0} A[2]$ (this is again contained in (1) of the proof of loc.cit.). Therefore for all $y \in A$ we have the decomposition as direct sum of 1-dimensional subspaces

$$
\bigoplus_{\alpha \in \operatorname{Pic}^{0} A[2]} H^{0}\left(\mathcal{E} \otimes L^{-1} \otimes \alpha\right) \otimes \alpha(y) \cong\left(\mathcal{E} \otimes L^{-1}\right)(y)
$$

The commutative diagram

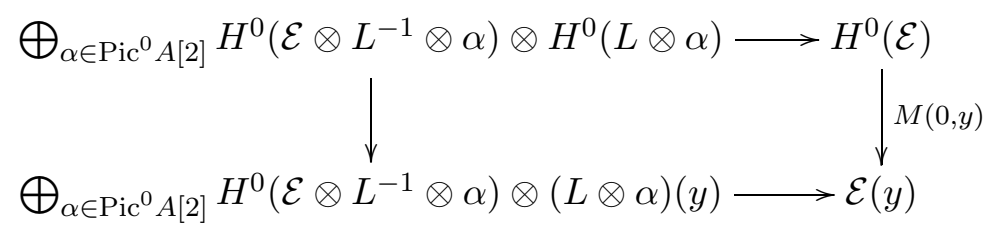

shows that the rank of the map $M(0, y)$ is precisely the number of line bundles $\alpha \in \operatorname{Pic}^{0} A[2]$ such that the evaluation of $H^{0}(L \otimes \alpha)$ at $y$ is non-zero. One gets the statement of Theorem 2.1 via the isomorphism $\varphi_{L}: A \rightarrow \operatorname{Pic}^{0} A$ sending $x$ to $\left(t_{x}^{*} L\right) \otimes L^{-1}$.

\section{Proof of Theorem 1.1}

3.1. We keep the notation introduced in the previous section. Specifically, let $L=\mathcal{O}_{A}(\Theta)$, where $\Theta$ is a symmetric theta divisor. For a point $x \in A$ we denote $V_{x}$ the image of the multiplication map $M(0, x)$. For all $x, y \in A$ let us consider the following commutative diagram of multiplication maps of global sections

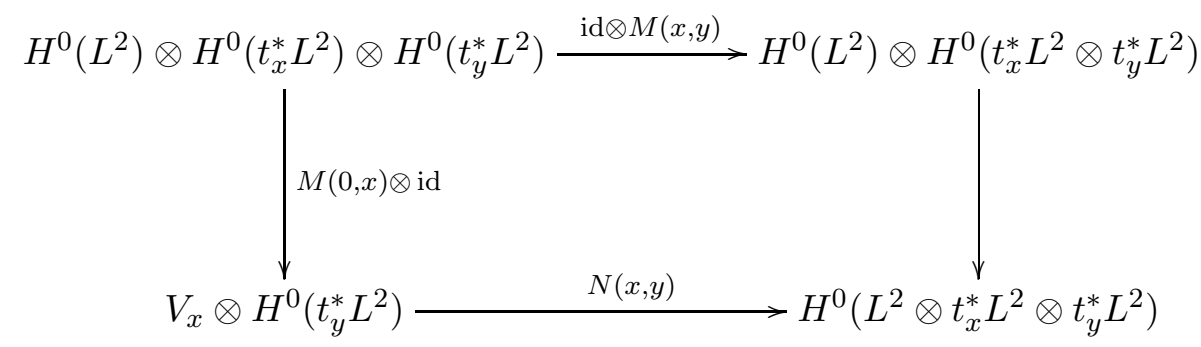

By Theorem 2.3 the right vertical arrow is surjective for all $x, y \in A$, since, by the theorem of the square, $t_{x}^{*} L^{2} \otimes t_{y}^{*} L^{2}=\left(t_{z}^{*} L\right)^{4}$ for a suitable $z$. Let us fix a point $x \in A$. By Theorem 2.2 the map id $\otimes M(x, y)$ is surjective for general $y \in A$. Therefore, for such $y$ 's, the map $N(x, y)$ is surjective, 
hence $\operatorname{dim} V_{x} \cdot 2^{g} \geq 6^{g}$. In conclusion for all $x \in A$ the rank of the map $M(0, x)$ is $\geq 3^{g}$. Therefore, by Theorem 2.1

$$
(\Theta+x)(2) \leq 4^{g}-3^{g} .
$$

for all $x \in A$. Since every divisor representing the principal polarization is a translate of a symmetric one, this proves the first assertion of Theorem 1.1.

3.2. To prove the last assertion we need to construct a commutative diagram of locally free sheaves on $A$ inducing diagram (3.1) at the fiber level. To this purpose we consider a Poincaré line bundle $\mathcal{P}$ on $A \times \operatorname{Pic}^{0} A$. Given a coherent sheaf $\mathcal{F}$ such that $h^{i}\left(\mathcal{F} \otimes P_{\alpha}\right)=0$ for all $i>0$ and for all line bundles $\alpha \in \operatorname{Pic}^{0} A$ we denote $\Phi(\mathcal{F})$ the coherent sheaf on $\operatorname{Pic}^{0} A$ defined as follows:

$$
\Phi(\mathcal{F})=q_{*}\left(p^{*}(\mathcal{F}) \otimes \mathcal{P}\right)
$$

where $p$ and $q$ are respectively the first and second projection of $A \times \operatorname{Pic}^{0} A[2$ By base change the hypothesis on $\mathcal{F}$ yields that $\Phi(\mathcal{F})$ is a locally free sheaf whose fibre at the point $\alpha \in \operatorname{Pic}^{0} A$ is canonically identified to the vector space $H^{0}(A, \mathcal{F} \otimes \alpha)$.

For $x \in A$ we consider the commutative diagram of locally free sheaves on $\operatorname{Pic}^{0} A$

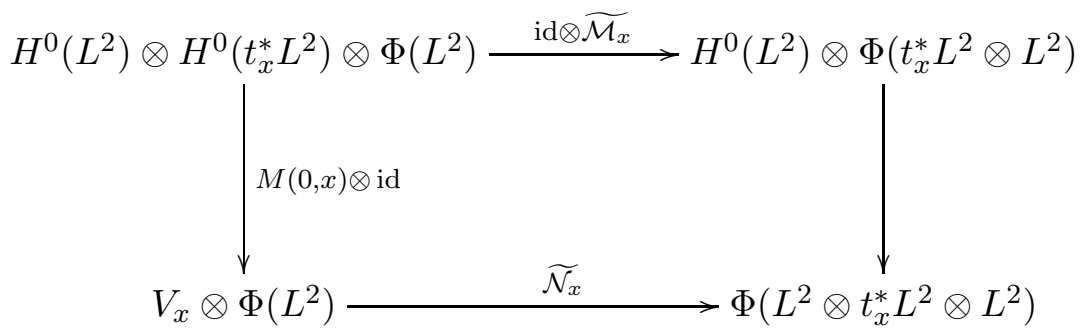

where the maps $\widetilde{M_{x}}, \widetilde{N_{x}}$ and the left vertical arrow are fiberwise multiplication maps of global sections. The definition of the maps appearing in diagram (3.2) is left to the reader.

For an ample line bundle $M$ on $A$ let us denote $\varphi_{M}: A \rightarrow \operatorname{Pic}^{0} A$ the isogeny associated to the polarization represented by $M: \varphi_{M}(y)=\left(t_{y}^{*} M\right) \otimes M^{-1}$. As $L$ represents a principal polarization, $\varphi_{L}: A \rightarrow \mathrm{Pic}^{0} A$ is an isomorphism. Via such isomorphism, for all positive integers $k$ the isogeny $\varphi_{L^{k}}$ is identified to the multiplication by $k$ homomorphism $k_{A}: A \rightarrow A$, defined by $y \mapsto k y$. Applying $\varphi_{L^{2}}^{*}$ to diagram (3.2) we get

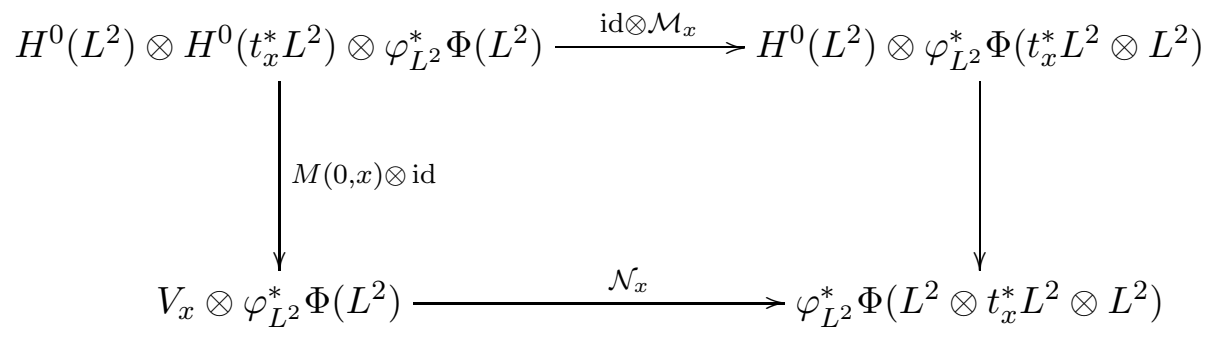

By construction diagram (3.3) induces diagram (3.1) at the fibre level.

\footnotetext{
${ }^{2}$ Thanks to the hypothesis on $\mathcal{F}$ this is in fact the Fourier-Mukai transform of $\mathcal{F}$.
} 
3.3. Next, we make some calculations to compute the (determinant of) the locally free sheaves appearing in diagram (3.3). It is well known ([14, Prop. 3.11]) that, for an ample line bundle $M$ on $A$,

Therefore

$$
\varphi_{M}^{*} \Phi(M) \cong H^{0}(M) \otimes M^{-1}
$$

$$
\varphi_{L^{2}}^{*} \Phi\left(L^{2}\right) \cong H^{0}\left(L^{2}\right) \otimes L^{-2}
$$

Similarly, since the isogeny $\varphi_{L^{k}}$ is identified to the isogeny $k_{A}$, we have that

$$
\begin{gathered}
\left.2_{A}^{*}\left(\varphi_{L^{2}}^{*} \Phi\left(t_{x}^{*} L^{2} \otimes L^{2}\right)\right) \cong H^{0}\left(t_{x}^{*} L^{2} \otimes L^{2}\right)\right) \otimes t_{-x}^{*} L^{-2} \otimes L^{-2} \\
\left.3_{A}{ }^{*}\left(\varphi_{L^{2}}^{*} \Phi\left(L^{2} \otimes t_{x}^{*} L^{2} \otimes L^{2}\right)\right) \cong H^{0}\left(L^{4} \otimes t_{x}^{*} L^{2}\right)\right) \otimes L^{-4} \otimes t_{-x}^{*} L^{-2}
\end{gathered}
$$

Since, given a line bundle $M$ on $A$, the line bundle $k_{A}^{*} M$ is algebraically equivalent to $M^{k^{2}}$, after short calculations we get

$$
\begin{gathered}
\operatorname{det}\left(\varphi_{L^{2}}^{*} \Phi\left(L^{2}\right) \sim L^{-2^{g+1}}\right. \\
\operatorname{det}\left(\varphi_{L^{2}}^{*} \Phi\left(t_{x}^{*} L^{2} \otimes L^{2}\right) \sim L^{-4^{g}}\right. \\
\operatorname{det}\left(\varphi_{L^{2}}^{*} \Phi\left(L^{2} \otimes t_{x}^{*} L^{2} \otimes L^{2}\right)\right) \sim L^{-4 \cdot 6^{g-1}}
\end{gathered}
$$

where $\sim$ means algebraic equivalence.

3.4. By Theorem 2.2 the map id $\otimes \mathcal{M}_{x}$ drops rank at a divisor $2^{g} E_{x}$ where $E_{x}$ is a divisor such that

$$
\operatorname{supp}\left(E_{x}\right)=\operatorname{supp}\left(\sum_{\eta \in A[2]} \Theta+x+\eta\right)
$$

By (3.4) and (3.5) $E_{x} \sim 4^{g} \Theta$ hence

$$
E_{x}=\sum_{\eta \in A[2]} \Theta+\eta+x
$$

3.5. After this preparation now we are ready for the proof of the second part of Theorem 1.1. To begin with we claim that if $\geq 2$ and $\Theta$ is irreducible then the bound of Theorem 1.1 cannot be achieved, i.e. $\operatorname{dim} V_{x}>3^{g}$ for all $x \in A$. Indeed, if $\operatorname{dim} V_{x}=3^{g}$ for a point $x \in A$ then the two locally free sheaves of the bottom arrow of diagram (3.3) have the same rank, so that the map drops rank on a divisor $D_{x}$. From (3.4) and (3.6) it follows that

$$
D_{x} \sim 8\left(6^{g-1} \Theta\right)
$$

By Theorem 2.3 the support of $D_{x}$ is contained in the support of $E_{x}$. However, nothing changes if we replace $L$ with $t_{\eta}^{*} L$, with $\eta \in A[2]$. Therefore both the divisors $D_{x}$ and $E_{x}$ are invariant with respect to the action of $A[2]$. Hence

$$
\operatorname{supp}\left(E_{x}\right)=\operatorname{supp}\left(D_{x}\right)=\sum_{\eta \in A[2]} \Theta+\eta+x
$$

In conclusion, if $\Theta$ is irreducible

$$
\operatorname{supp}\left(D_{x}\right)=\sum_{\eta \in A[2]} \Theta+\eta+x
$$

Hence $D_{x}$ must be, up to algebraic equivalence, an integral multiple of $4^{g} \Theta$. But this, for $g \geq 3$, is clearly in contrast with (3.7). Concerning the case $g=2$, one can prove directly that $\Theta$ is 
irreducible then for all $x \in A$ the rank of the map $M(0, x)$ is $>9$. If $x \in A[2]$ this is well known, see [6] (recall that $M(0,0)=M(0, x)$ for all $x \in A[2]$ ). The same thing should be known also in the case $x \notin A[2]$. However, for lack of reference, we include a proof (of a more precise statement) in Lemma 3.1 below. Therefore, as claimed, assuming $g \geq 2$, if for some $x \in A$ the rank of the map $M(0, x)$ is equal to $3^{g}$ then $\Theta$ must be reducible. Finally, for sake of completeness, we recall that for an elliptic curve $A$ the rank of the map $M(0, x)$ is equal to 3 if and only if $x \in A[2]$.

3.6. By the previous step and the the decomposition theorem it follows that if, for some $x \in A$, $(\Theta+x)(2)=4^{g}-3^{g}$ then the principally polarized abelian variety $A$ is the product of two lower dimensional principally polarized abelian varieties. At this point, arguing by induction, we have that $A$ is a product of elliptic curves and

$$
E_{x}=4^{g-1} H, \quad D_{x}=6^{g-1} 2 H
$$

where

$$
H=\sum_{\substack{x_{i} \in E_{i}[2] \\ i=1, \ldots, g}} E_{1} \times \cdots \times\left\{x_{i}\right\} \times \cdots \times E_{g} .
$$

Theorem 1.1 follows.

Lemma 3.1. Let $(A, \Theta)$ be a principally polarized abelian variety of dimension 2 with $\Theta$ irreducible. Then, keeping the above notation, $\operatorname{rkM}(0, x) \geq 11$ if $x \notin A[2]$.

Proof. Since $\Theta$ is irreducible, it is a smooth curve of genus 2, say $C$. Keeping the above notation we have the following exact sequences

$$
\begin{gathered}
0 \rightarrow H^{0}(L) \rightarrow H^{0}\left(L^{2}\right) \rightarrow H^{0}\left(L_{\mid C}^{2}\right) \rightarrow 0 \\
0 \rightarrow H^{0}\left(L^{-1} \otimes t_{x}^{*} L^{2}\right) \rightarrow H^{0}\left(t_{x}^{*} L^{2}\right) \rightarrow H^{0}\left(\left(t_{x}^{*} L^{2}\right)_{\mid C}\right) \rightarrow 0
\end{gathered}
$$

inducing the filtration on the tensor product of the middle vector spaces

$$
H^{0}(L) \otimes H^{0}\left(L^{-1} \otimes t_{x}^{*} L^{2}\right) \subset U \subset H^{0}\left(L^{2}\right) \otimes H^{0}\left(t_{x}^{*} L^{2}\right)
$$

where

$$
\frac{U}{H^{0}(L) \otimes H^{0}\left(L^{-1} \otimes t_{x}^{*} L^{2}\right)} \cong\left(H^{0}(L) \otimes H^{0}\left(\left(t_{x}^{*} L^{2}\right)_{\mid C}\right)\right) \oplus\left(H^{0}\left(L^{-1} \otimes t_{x}^{*} L^{2}\right) \otimes H^{0}\left(L_{\mid C}^{2}\right)\right)
$$

and

$$
\frac{H^{0}\left(L^{2}\right) \otimes H^{0}\left(t_{x}^{*} L^{2}\right)}{U} \cong H^{0}\left(L_{\mid C}^{2}\right) \otimes H^{0}\left(\left(t_{x}^{*} L^{2}\right)_{\mid C}\right)
$$

We have also the filtration

$$
H^{0}\left(t_{x}^{*} L^{2}\right) \subset H^{0}\left(L \otimes t_{x}^{*} L^{2}\right) \subset H^{0}\left(L^{2} \otimes t_{x}^{*} L^{2}\right)
$$

where obviously

$$
\frac{H^{0}\left(L \otimes t_{x}^{*} L^{2}\right)}{H^{0}\left(t_{x}^{*} L^{2}\right)} \cong H^{0}\left(L \otimes t_{x}^{*} L_{\mid C}^{2}\right) \quad \text { and } \quad \frac{H^{0}\left(L^{2} \otimes t_{x}^{*} L^{2}\right)}{H^{0}\left(L \otimes t_{x}^{*} L^{2}\right)} \cong H^{0}\left(L^{2} \otimes t_{x}^{*} L_{\mid C}^{2}\right)
$$

The multiplication map of global sections $M(0, x)$ is in fact a map of filtered vector spaces from (3.8) to (3.9). Since $x \notin A[2]$, the line bundles $L_{\mid C}^{2}$ and $\left(t_{x}^{*} L^{2}\right)_{\mid C}$ do not coincide. This being the case the multiplication map of global sections $H^{0}\left(L_{\mid C}^{2}\right) \otimes H^{0}\left(\left(t_{x}^{*} L^{2}\right)_{\mid C}\right) \rightarrow H^{0}\left(\left(L^{2} \otimes t_{x}^{*} L^{2}\right)_{\mid C}\right)$ is surjective, hence of rank 7 . This could be proved directly, however it is a (very) particular case 
of a general result of Eisenbud, Koh and Stillman about multiplication maps of global sections on curves, and even syzygies, see [9, Thm 2]. On the other hand, we claim that the induced map $U \rightarrow H^{0}\left(L \otimes t_{x}^{*} L^{2}\right)$ has rank $\geq 4$. Therefore the rank of the map $M(0, x)$ is $\geq 11$, proving the Lemma. To prove the claim, note that the map $H^{0}(L) \otimes H^{0}\left(L^{-1} \otimes t_{x}^{*} L^{2}\right) \rightarrow H^{0}\left(t_{x}^{*} L^{2}\right)$ has rank 1 and the map

$$
\left(H^{0}(L) \otimes H^{0}\left(\left(t_{x}^{*} L^{2}\right)_{\mid C}\right)\right) \oplus\left(H^{0}\left(L^{-1} \otimes t_{x}^{*} L^{2}\right) \otimes H^{0}\left(L_{\mid C}^{2}\right)\right) \rightarrow H^{0}\left(\left(L \otimes t_{x}^{*} L^{2}\right)_{\mid C}\right)
$$

has rank 3. Indeed, while the restriction to the first summand is zero, the restriction to the second summand is injective. Indeed, since $x \notin A[2], L^{-1} \otimes t_{x}^{*} L^{2}$ is not isomorphic to $L$, the restriction to $C$ of the 1-dimensional space of global sections $H^{0}\left(L^{-1} \otimes t_{x}^{*} L^{2}\right)$ is non-zero.

\section{VARIANTS, QUESTIONS AND REMARKS}

4.1. We start with a remark concerning Theorem 1.1. We observe that only when $A$ is a product of elliptic curves and $\Theta$ is a symmetric theta divisor of $A$ the map induced by $|2 \Theta|$ is of degree $2^{g}$ and the image is smooth. In fact it is, up to a projectivity, the image of the Veronese map of $g$ copies of $\mathbb{P}^{1}$ into $\mathbb{P}^{2^{g}-1}$. In this case the image is defined by the intersection of $2^{g-1}\left(2^{g}+1\right)-3^{g}$ quadrics that are a basis of the kernel of the map

$$
\operatorname{Sym} M(0,0): \operatorname{Sym}^{2} H^{0}\left(A, L^{2}\right) \rightarrow H^{0}\left(A, L^{4}\right)
$$

4.2. Theorem 1.1 yields a (non-optimal) bound for the numbers $\Theta(n)$ for $n=2 m$ even, when the characteristic of the field does not divide $n$. In fact, since $A[2 m] / A[2] \equiv(\mathbb{Z} / m \mathbb{Z})^{2 g}$, Theorem 1.1 implies

\section{Corollary 4.1.}

$$
\Theta(2 m) \leq m^{2 g}\left(4^{g}-3^{g}\right)
$$

For $n$-torsion points the bound should be $n^{2 g}-\left(n^{2}-1\right)^{g}$, with equality if and only if $(A, L)$ is the polarized product of elliptic curves, cf.[1]. A certain evidence for this can be deduced from Theorem 2.2. In fact we know that $M(0, y)$ is not surjective if and only if

$$
y \in \bigcup_{\eta \in A[2]} \Theta+\eta
$$

In particular the multiplication map $M(0, y)$ is surjective for general $y \in A$.

Now assume that $y$ is a $n=2 m$ torsion point, thus we have

$$
\Theta(n)=\bigoplus_{y \in A[n] / A[2]} \operatorname{dim} \operatorname{ker}(M(0, y))
$$

When $n>>0$, because of the density of torsion points, in mostly of cases we have that the dimension is 0 . Hence we can improve the result of Corollary 4.1 with the following estimate

$$
\Theta(n) \leq\left(4^{g}-3^{g}\right) K_{n} m^{2 g-2}
$$

for a suitable constant $K_{n}$. 
4.3. In the proof of Theorem 1.1 an important role is played by the multiplication maps of global sections $M(0, y): H^{0}\left(A, L^{2}\right) \otimes H^{0}\left(A, t_{y}^{*} L^{2}\right) \rightarrow H^{0}\left(A, L^{2} \otimes t_{y}^{*} L^{2}\right)$, where $L$ represents the principal polarization. A crucial aspect of such maps is that dimensions of the source and of the target are equal. This doesn't happen for $n$ higher than 2. However in the paper [8] natural analogues of the above maps were introduced. These are the "fractional" multiplication maps of global sections

$$
H^{0}\left(A, L^{n}\right) \otimes H^{0}\left(A, t_{y}^{*} L^{n(n-1)}\right) \rightarrow H^{0}\left(A,(n-1)_{A}^{*}\left(L^{n}\right) \otimes t_{y}^{*} L^{n(n-1)}\right)
$$

obtained by factoring with the natural inclusion of the first factor into $H^{0}\left(A,(n-1)_{A}^{*} L^{n}\right)$, where $(n-1)_{A}: A \rightarrow A$ is the isogeny $x \mapsto(n-1) x$. We refer to the discussion after the proof of Corollary 8.2 of [8] for some explanation of the attribute "fractional" as well as why such maps play the same role of the maps $M(0, y)$ for higher $n$. At present we are not able to treat them as we do for "integral" multiplication maps of global sections, but we hope to come back to this in the future.

4.4. Next, we notice that, in the complex case, in the paper of Marcucci and Pirola, Theorem 1.1 yields a lower bound on the number of non-effective square roots of line bundles of degree $\leq g-1$ on curves of genus $g$, as well as related bounds (see [13, Prop. 2.1]).

4.5. It would be nice to get an estimate of $\Theta(2)$ when $(A, \Theta)$ is an irreducible principally polarized abelian variety. At the best of our knowledge, in the complex case, we have that if $(A, \Theta)$ is the jacobian of a hyperelliptic curve and $\Theta$ is symmetric thus

$$
\Theta(2)=4^{g}-\left(\begin{array}{c}
2 g+1 \\
g
\end{array}\right)
$$

This is a well known result, cf. [15]. It seems natural to guess that this is the maximal number (see also [19]). In other known examples, it appears fundamental the existence of an involution, cf. 2 and 7]. Nevertheless in genus 4 there is an irreducible principally polarized abelian variety that is not a jacobian with $\Theta(2)=130$, the same as the hyperelliptic case (see [22] or [5]). In this case the period matrix is related to the Gaussian lattice $E_{8}$. In higher dimension this class of examples have a number of vanishing thetanulls which is smaller compared to the hyperelliptic Jacobians. However, as pointed out in [3], in a particular subcase, these vanishing thetanulls have the particular property of being syzygetic (in the classical terminology). It would also be interesting to get a bound for this special case.

\section{REFERENCES}

[1] R. Auffarth, G. P. Pirola, R. Salvati Manni Torsion points on theta divisors, Proc. Amer. Math. Soc. 145 (2017), 89-99; arXiv:1512.09296

[2] A. Beauville Vanishing thetanulls on curves with involution Rendiconti del Circolo Matematico di Palermo, 62 (2013), 61-66

[3] A. Beauville Abelian varieties associated to Gaussian lattices A Celebration of Algebraic Geometry, Clay Mathematics Proceedings 18 (2013), 37-44

[4] F. Dalla Piazza, A. Fiorentino, S. Grushevsky, S. Perna, R. Salvati Manni, Vector-valued modular forms and the Gauss map, Documenta Mathematica 22 (2017), 1063-1080.

[5] O. Debarre Annulation de thetaconstantes sur les variétés abéliennes de dimension quatre C.R. Acad. Sci. Paris 305 (1987) 885-888

[6] E. Freitag Zur Theorie der Modulformen zweiten Grades. Nachr. der Akad.d.Wiss., Gttingen (1965) 151157

[7] B. van Geemen Projective models of Picard modular varieties in Classification of Irregular Varieties, Springer LNM 1515

[8] Z. Jiang, G. Pareschi Cohomological rank functions on abelian varieties, preprint (2017) to appear in Annales ENS 
[9] D. Eisenbud, J. Koh, M. Stillman Determinantal equations for curves of high degree, Amer. J. of Math. 110 (1988) 513-539

[10] G. Kempf. Complex Abelian Varieties and Theta Functions, Springer 1991.

[11] G. Kempf, Multiplication Over Abelian Varieties. Amer. J. of Math. 110 (1988) pp. 765-773

[12] S. Koizumi, Theta relations and projective normality of abelian varieties, Amer. J. of Math. 98 (1976) 865-889

[13] V. Marcucci, G. Pirola. Points of order 2 on theta divisors, Atti Accad. Naz. Lincei Cl. Sci. Fis. Mat. Natur. Rend. Lincei (9) Mat. Appl. 23 (2012) 319-323.

[14] S. Mukai, Duality between $D(X)$ and $D(\widehat{X})$ with its application to Picard sheaves, Nagoya Math. J. 81 (1981) 153-175

[15] D. Mumford Tata Lectures on Theta II, Birkäuser 1984

[16] A. Ohbuchi, A note on the normal generation of ample line bundles on abelian varieties, Proc. Japan Acad. 64 (1988), 119-120

[17] G. Pareschi, Syzygies of abelian varieties, Journal of the A.M.S. 13 (2000), 651-664

[18] G. Pareschi and M. Popa, Regularity of abelian varieties II: basic results on linear series and defining equations, J. Alg. Geom. 13 (2004) 167-193.

[19] C. Poor, The hyperelliptic locus, Duke Math. J. 76 (1994), no. 3, 809-884.

[20] R. Salvati Manni, Modular varieties with level 2 theta structure, Amer. J. Math. 116 (1994) $1489-1511$.

[21] T. Sekiguchi. On the cubics defining abelian varieties, J. Math. Soc. Japan 30 (1978) 703-721.

[22] R. Varley Weddle's Surfaces, Humbert's Curves, and a Certain 4-Dimensional Abelian Variety, Amer. J. Math. 108(1986), 931-951

G. Pareschi, Dipartimento di Matematica, Università di Tor Vergata, italy

E-mail address: pareschi@mat.uniroma2.it

R. Salvati Manni, Dipartimento di Matematica "Guido Castelnuovo", Università di Roma "La SAPIENZA", ITALY

E-mail address: salvati@mat.uniroma1.it 